\title{
Coated NPK Fertilizer Based on Citric Acid-Crosslinked Chitosan/Alginate Encapsulant
}

\author{
Vince St D. Mesias' ${ }^{1}$ Anne Bernadette S. Agu', Precious Japheth L. Benablo', \\ Chun-Hu Chen², David P. Penaloza Jr. ${ }^{*}$ \\ 1 Chemistry Department, College of Science, De La Salle University, 2401 Taft Avenue, Manila 0922, Philippines \\ 2 Department of Chemistry, National Sun Yat-sen University, Kaohsiung, Taiwan \\ * Corresponding author's email: david.penaloza.j@@dlsu.edu.ph
}

\begin{abstract}
A coated nitrogen-phosphorus-potassium (NPK) fertilizer was prepared via crosslinking chitosan and alginate using citric acid. Fourier transform infrared (FTIR) spectroscopy, scanning electron microscopy (SEM), particle size analysis, and zeta potential measurement showed the successful crosslinking, appropriate size, and colloidal stability of the coated NPK fertilizer. The encapsulation capability of the crosslinked chitosan/alginate (Chi/Alg) was studied using fluorescence spectroscopy and NPK analysis. The release behavior studies under various $\mathrm{pH}$ conditions showed that Chi/Alg NPK conformed to the standards of controlled release fertilizer with a maximum release rate of $40 \%$ for 30 days. The investigation of the release mechanism using Korsemeyer-Peppas mathematical model showed that the release of nutrients is governed by both coating material relaxation and diffusion process.
\end{abstract}

Keywords: controlled release, crosslinking, encapsulation, NPK fertilizer

\section{INTRODUCTION}

The nanostructured materials with novel properties that can be easily fine-tuned are ideal to make agriculture-based smart systems. Such smart materials, including encapsulation and controlled release systems, have revolutionized the agrochemical industries by providing more effective delivery of fertilizers (Azeem, KuShaari, Man, Basit, \& Thanh, 2014; Jarosiewicz \& Tomaszewska, 2003) and pesticides (Campos, de Oliveira, Fraceto, \& Singh, 2015; Roy, Singh, Bajpai, \& Bajpai, 2014) resulting in the enhanced nutrient absorption, lower loss of the active ingredient (Dasgupta, Madras, \& Chatterjee, 2016; Roy, et al., 2014) - thereby lowered possibility of leaching to the environment (more environmentfriendly). These technologies can be further designed in such ways that they can respond to environmental changes- such systems are called smart materials (Ni, Liu, Lü, Xie, \& Wang, 2011; Xiao, Zhou, \& Qian, 2017). Generally, the controlled release fertilizers cost more than the conventional (uncoated) fertilizers, such as urea. A controlled release fertilizer using natural polymers can provide a cheap but efficient nutrient management strategy (Azeem, et al., 2014; Ni, et al., 2011), not only because it can release the nutrient contents gradually and minimize the environmental pollution - but also the natural polymers abound in nature (Hu \& Huang, 2013; Krishna Sailaja, Amareshwar, \& Chakravarty, 2011; RodríguezVelázquez, Alatorre-Meda, \& Mano, 2015) These biopolymers, especially in the form of hydrogels, are great candidate materials for the creation of encapsulation systems (Brown \& McShane, 2006; Habibi, Kamaly, Memic, \& Shafiee, 2016; Hori, Penaloza, Shundo, \& Tanaka, 2012; Lima, Song, Blanco-Fernandez, Alvarez-Lorenzo, \& Mano, 2011; Qu, Zhao, Ma, \& Guo, 2017; Seiffert, Thiele, Abate, \& Weitz, 2010; Shundo, et al., 2014; Shundo, Penaloza, \& Tanaka, 2013; Wang, Papadimitrakopoulos, \& Burgess, 2013). The controlled release potential of hydrogels using crosslinked polymers is being explored for the environment-friendly and cost-effective coated 
fertilizer applications (Bortolin, et al., 2012; Bortolin, Aouada, Mattoso, \& Ribeiro, 2013; Essawy, Ghazy, El-Hai, \& Mohamed, 2016; Jamnongkan \& Kaewpirom, 2010; SaraydIn, Karadağ, \& Güven, 2000). As an example, chitosan hydrogel was utilized by Jamnongkan and Kaewpirom to study the release kinetics of potassium (Jamnongkan \& Kaewpirom, 2010).

While alginate is a preferred material for encapsulation applications (Das, Kasoju, \& Bora, 2010; Gombotz \& Wee, 1998; Rowley, Madlambayan, \& Mooney, 1999; Sultana, et al., 2000; Tan \& Takeuchi, 2007), it is highly soluble in water, limiting its role as an encapsulating material. Such issue of instability can be addressed by crosslinking (Awadhiya, Kumar, \& Verma, 2016; Demitri, et al., 2008; Monteiro \& Airoldi, 1999; Oryan, Kamali, Moshiri, Baharvand, \& Daemi, 2018). Chitosan, on the other hand, is also a biopolymer of interest. It has been studied thoroughly as an active ingredient carrier since it is biodegradable, cost-effective, and less toxic (Dash, Chiellini, Ottenbrite, \& Chiellini, 2011; Y. Liu, Shen, Zhou, Wang, \& Deng, 2016; Stulzer, Tagliari, Parize, Silva, \& Laranjeira, 2009; Zhao, Yim, Du, \& Abu-Lebdeh, 2018). Chitosan could be derived from the crustacean waste material and the possible source would be its industrial waste, which reduces the costs (Stulzer, et al., 2009).

In solution, chitosan has been found to form a complex with negatively charged polymers due to its cationic character, this overcomes the limitation of alginate as an encapsulant (Araújo, Romão, Doumer, \& Mangrich, 2017; Picone \& Cunha, 2013; Stulzer, et al., 2009). The combination of chitosan and alginate, after being subjected to crosslinking using citric acid, could result in a more effective controlled release performance, compared to chitosan or alginate alone (Baysal, Aroguz, Adiguzel, \& Baysal, 2013; Kumar, Chauhan, Gopal, Kumar, \& Dilbaghi, 2015; Li, Dai, Zhang, Wang, \& Wei, 2008; Zhang, Wei, Lv, Wang, \& Ma, 2011). Citric acid is considered as one of the important organic acids that has a wide commercialization potential. Recently, major production of citric acid was conducted via microbial fermentation, as it is economical and easy to handle. Thus, using citric acid will reduce the cost of the typical controlled release fertilizer (Awadhiya, et al., 2016; de Cuadro, et al., 2015; Demitri, et al., 2008; Ghorpade, Yadav, \& Dias, 2016; H. J. Kim, Koo, Kim, Hwang, \& Im, 2017; Y. Liu, et al., 2016; Mali, Dhawale, \& Dias, 2017; Ren, et al., 2018).
In this regard, this study involved the investigation of the controlled release property of the crosslinked NPK fertilizer that is formed from the crosslinking of the chitosan/alginate (Chi/ $\mathrm{Alg}$ ) using citric acid (CA) encapsulating NPK fertilizer - hereby referred to as Chi/Alg/CA/ NPK. Moreover, this study was able to determine the effect of the varying amounts of crosslinker (citric acid as the crosslinker) and polymer blend (Chi/Alg) ratio on the particle size and encapsulation efficiency. It also determined the controlled release behavior in vitro as well as through a pot experiment.

\section{EXPERIMENTAL}

\section{Materials}

Chitosan (low molecular weight, MW90,000 g/mol), sodium alginate (low molecular weight,MW-80,000 g/mol) glacial acetic acid $(\mathrm{AR}, 99.7 \mathrm{v} / \mathrm{v})$, citric acid monohydrate (AR, $99.5 \% \mathrm{w} / \mathrm{w})$, potassium dihydrogen phosphate (AR, 99.5\% w/w), calcium chloride dihydrate (AR, 99.5\% w/w), ammonium chloride (AR, 99.5\% w/w), $\mathrm{pH}$ buffers (AR 4.00, 7.00, 9.00), nitric acid (AR 69\% v/v), hydrochloric acid (AR, $37 \% \mathrm{v} / \mathrm{v}$ ), and sodium 8-anilino-1-naphthalenesulfonate (AR, 97\% w/w) were used. The deionized water was obtained from ELGA Pure Lab Deionizer at the Bureau of Soils and Water Management (BSWM) Laboratory.

\section{Preparation of the chitosan/alginate encapsulated NPK fertilizer}

The $3.0 \%(\mathrm{w} / \mathrm{v})$ solution of sodium alginate was prepared by dissolving appropriate amounts of polymer in deionized water with continuous stirring up to $2 \mathrm{~h}$, whereas the $3.0 \%(\mathrm{w} / \mathrm{v})$ chitosan solution was prepared by dissolving an appropriate amount of polymer in $0.1 \mathrm{M}$ acetic acid, followed by dilution with deionized water. Then, both solutions were heated at $70^{\circ} \mathrm{C}$ and followed by the addition of NPK fertilizers in the form of $\mathrm{NH}_{4} \mathrm{Cl}$ and $\mathrm{KH}_{2} \mathrm{PO}_{4}$ to the alginate solution. Subsequently, $10 \mathrm{~mL}$ of $2 \%(\mathrm{w} / \mathrm{v})$ $\mathrm{CaCl}_{2}$ solution was added to the alginate solution and was continuously stirred at room temperature. This was followed by the addition of citric acid as a crosslinker. Chitosan was then added dropwise to the alginate solution while being stirred at $1000 \mathrm{rpm}$. 


\section{Encapsulation efficiency of the coated chitosan/alginate (Chi/Alg) NPK fertilizer}

This determination of the encapsulation efficiency was based on the method described by Kumar and his colleagues published elsewhere (Kumar, et al., 2015) with some modifications. The colloidal dispersion was centrifuged at 10,000 rpm for 15 minutes; the residue was washed and filtered afterwards. The supernatant liquid was collected and added to the filtrate; then, it was diluted with deionized water and was subjected to the NPK analysis in terms of parts per million (ppm). The excess or uncoated mass of each element was calculated using total volume of liquid and concentration from the NPK analysis. Afterwards, the mass was subtracted to the total mass of NPK to obtain the mass encapsulated. Finally, the encapsulation efficiency was calculated as the ratio of the mass encapsulated and the total mass.

\section{Characterization methodologies employed}

The particle size distribution was performed to measure the average size of the synthesized particles and zeta potential for its stability in solution of each formulation was determined using Malvern ZetaSizer Nano ZS instrument. The samples were spun and filtered before pipetting into the cuvettes. The particle size was determined at a 90-degree angle using dynamic light scattering, while the zeta potential measurement utilized laser Doppler microelectrophoresis.

Fourier-transform infrared (FTIR) spectroscopy was utilized to monitor the reaction progress to prove the formation of the desired product. The FTIR spectra of the samples measured were obtained on a Thermo Scientific Class 1 Nicolet 6700 FTIR spectrometer in the range of $400-4000 \mathrm{~cm}^{-1}$ at 16 scans. Each of the samples tested was added with $\mathrm{KBr}$ and then pelletized prior to FTIR analysis. All samples from different stages of the crosslinking reaction were dried at $40^{\circ} \mathrm{C}$ for $12 \mathrm{~h}$. The different stages of the crosslinking process were as follows: the first portion was obtained from alginate-NPK mixture. The second was obtained by adding chitosan and the last one was obtained after the citric acid crosslinking at the end of the preparation process.

The encapsulation of a model encapsulant onto the matrix was investigated by fluorescence spectroscopy using Spectrum ASCII Fluorescence spectrometer. A fluorescent dye, sodium 8-anilino-1-naphthalenesulfonate (ANS), served as a model encapsulant molecule. A $50-\mathrm{mL}$ aliquot of the $5 \%(\mathrm{w} / \mathrm{v})$ dye dispersion was prepared and divided into three $15-\mathrm{mL}$ portions. The first portion contained only ANS dye, while the second and third were added with polymer blend (Chi/Alg) and crosslinked polymer blend (Chi/ $\mathrm{Alg} / \mathrm{CA}$ ), respectively. They were stirred for an hour at $700 \mathrm{ppm}$ then subjected to the aforementioned instrument.

The morphology of the crosslinked polymer system containing NPK fertilizer was characterized by scanning electron microscopy using JEOL 5300 Scanning Electron Microscope.

\section{Controlled release studies of the coated chitosan/alginate (Chi/Alg) NPK fertilizer under different $\mathrm{pH}$ conditions}

Three buffer solutions were used in the release study under various $\mathrm{pH}$ conditions: $\mathrm{pH}$ 5.0, 7.0, and 8.5. Exactly one gram polymer coated fertilizer (Chi/Alg/CA/NPK) formulation with the highest encapsulation efficiency was weighed and analyzed to determine the total content of NPK. Simultaneously, the same mass of polymer coated fertilizer was placed in each $100-\mathrm{mL}$ buffer solution, dispersed, and were kept at room temperature. The NPK released was examined at different times: $5,10,15,20$, and 30 days by taking 5-mL buffer portion from each container and diluting it to $50 \mathrm{~mL}$ with deionized water prior to the NPK chemical analysis. Lastly, the percent release was plotted against time of each nutrient under various $\mathrm{pH}$ conditions and the release kinetics of the Chi/Alg/CA/NPK fertilizers was investigated using the Korsemeyer-Peppas equation.

\section{Fertilizer efficacy evaluation of the coated chitosan/alginate (Chi/Alg) NPK fertilizer under different $\mathrm{pH}$ conditions}

This involved three setups: (A) untreated; (B) with uncoated NPK fertilizer (Chi/Alg/NPK); (C) with coated NPK fertilizer (Chi/Alg/CA/NPK). Each setup was prepared in triplicates, where approximately $700 \mathrm{~g}$ loam soil was placed in each pot. Then, a soil sample extracted in each pot was subjected for total NPK analysis to ensure that they have a comparable nutrient content. The 
mung bean seeds were swelled in water overnight. After noticeable enlargement of the seeds, five seeds were placed in each plot and observed for the germination process. After two days of sprouting, it was followed by the application of respective treatments. Finally, all pots were placed under direct sunlight and observed for growth. Plant height, leaf height and leaf width - all measurements were in $\mathrm{cm}$. All of these growth parameters were obtained weekly up to eight weeks and the fruiting time was also noted for each setup.

\section{RESULTS AND DISCUSSION}

In general, the NPK fertilizer dissolution is necessary in order for the particle distribution to occur resulting to a better encapsulation upon crosslinking (Ma, Tan, Dai, \& Zhou, 2013). However, modification was done because the presence of more water would reduce the encapsulation due to the NPK fertilizer solubility in aqueous solution, as some of them will escape the polymer crosslinking process (Ren, et al., 2018). The heating process is needed for the citric acid crosslinking mechanism to form ester bonds with hydroxyl groups of polymers at about $80^{\circ} \mathrm{C}$ (Demitri, et al., 2008), thus maximizing encapsulation process. Subsequently, the $\mathrm{Ca}^{+2}$ ions were introduced to induce the ionic gelation, then followed by polyelectrolyte complexation of chitosan and alginate which adds up to the encapsulating capacity of the polymer mixture (Kumar, et al., 2015). Continuous stirring was done under heating for the citric acid crosslinking as well as to increase the gelling while preventing particle growth of resulting in colloidal dispersion.

\section{Encapsulation efficiency of the coated chitosan/alginate (Chi/Alg) NPK fertilizer in various formulations}

The encapsulation efficiency of various Chi/ $\mathrm{Alg} / \mathrm{CA} / \mathrm{NPK}$ formulations obtained from varying the composition of polymers and amount of citric acid (CA) crosslinker is presented in Figure 1. It is clearly seen in the figure that an increase in CA has significantly increased the encapsulation efficiency of Chi/Alg. This is because crosslinking introduces a three-dimensional network structure (Chen, Gao, Qiu, \& Hu, 2013) that will entrap the substance in the polymer matrix (Shi, et al., 2008). Furthermore, when the amount of crosslinker was maintained at a constant level, the composition of the polymer blends has a significant effect on the encapsulation capacity. In Figure 1, variations of 1:2 and 2:1 have a smaller efficiency compared to $1: 1$, this is due to the fact that the imbalance of interaction caused by the dominance of one component results in less organized network which compromises the enhanced property created by combining two polymers (Riyajan \& Nuim, 2013). It was noted that the higher ratio of alginate (1:2) gives a higher efficiency relative to 2:1 formulations, since alginate performs more interactions with calcium ions (Kumar, et al., 2015) due to its sugar acid components giving carboxylate ions.

\section{Particle size analysis of the coated chitosan/ alginate (Chi/Alg)NPK fertilizer in various formulations}

For the Chi/Alg/CA/NPK formulations, it is shown in Figure 2 that at constant crosslinker concentration, the imbalanced interaction given by $1: 2$ and $2: 1$ composition results in a larger size

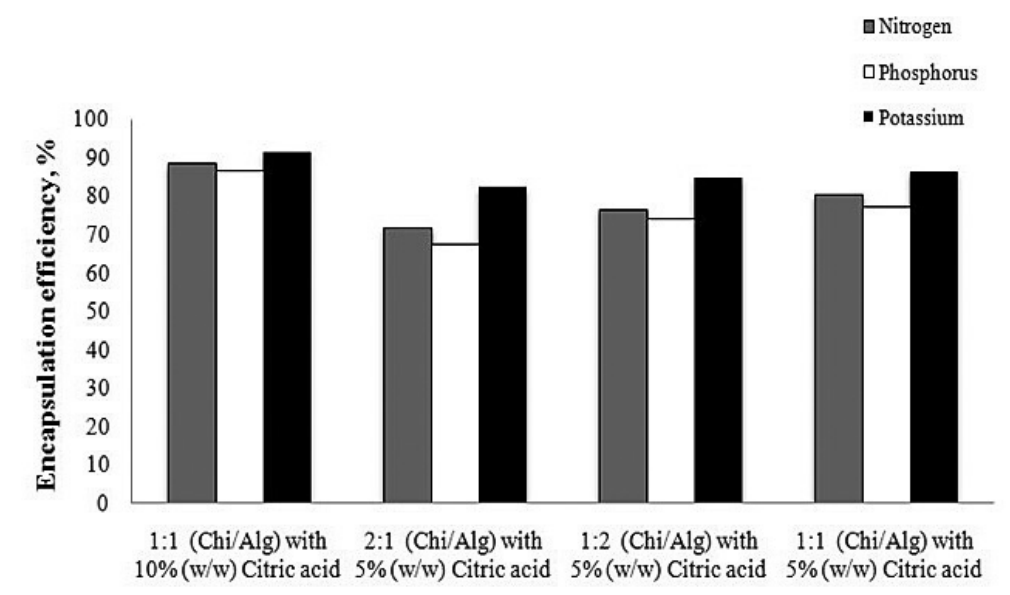

Figure 1. Encapsulation efficiency of various Chi/Alg/CA/NPK formulations. 
because of the attractive forces given by oppositely charged polymers, chitosan and alginate as they will form a more compact structure (Kumar, et al., 2015) resulting in smaller size like that of the 1:1 Chi/Alg formulation. Moreover, in the case of the Chi/Alg matrix, the excess alginate will form more gels with the $\mathrm{Ca}^{2+}$ ions, thus the average size is shifted to higher value. Generally, citric acid increases the size of the particle because of its crosslinking mechanism as described by Demitri and co-workers (Demitri, et al., 2008) and Riyajan and Nuium (Riyajan \& Nuim, 2013).

\section{Zeta potential measurements of the coated chitosan/alginate (Chi/Alg) NPK fertilizer in various formulations}

By observation, as in the case of Chi/Alg, the particles settled after about three days. Figure 3 supports the observation, the values lie above the $-30 \mathrm{mV}$, which means that particles will settle in a certain period of time. The reason for low zeta potential values is the dominance of van der Waals interaction over electrostatic repulsion (Zhang, et al., 2011). In reference to the preparation method, the concentration of the polymers were 3\% (w/v); hence, the higher amounts of polymer will introduce more van der Waals interaction overcoming the effect of electrostatic repulsion, since more particles are forming and the distance between them reduces. It can be observed from Figure 3 that the magnitude of zeta potential reduces as the amount of crosslinker is increased from $5 \%$ to $10 \%(\mathrm{w} / \mathrm{v})$ of $1: 1$ formulations. That is accounted for in the formation of organized network upon crosslinking (Maitra \& Shukla, 2014) and promotes more van der Waals interaction over electrostatic repulsion (Zhang, et al., 2011) leading to the aggregation of particles. In general, the sign of zeta potential dictates the dominant ionic charge in the surface. The negative values obtained in this study are reasonable,

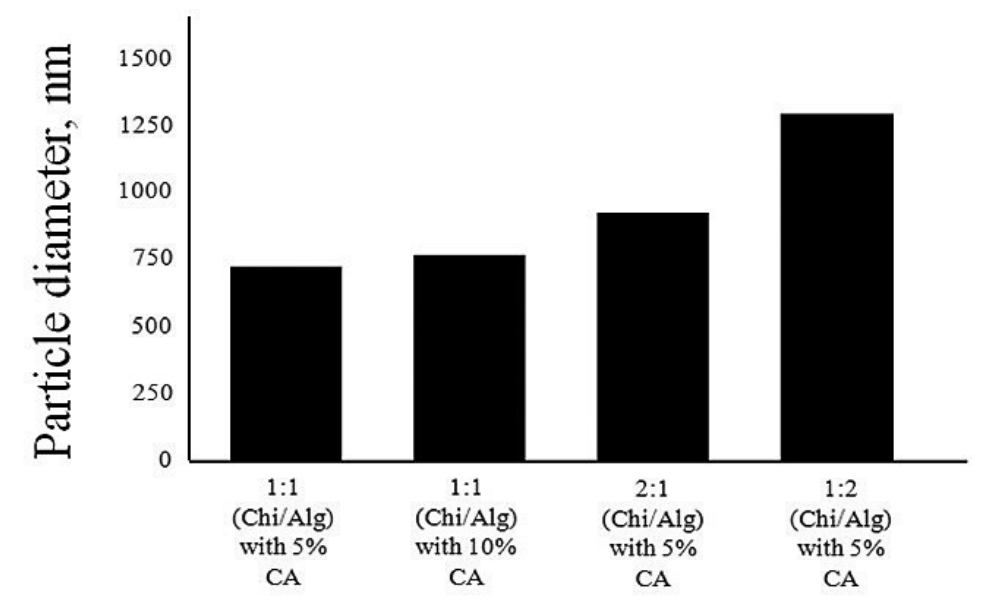

Figure 2. Average particle size of various $\mathrm{Chi} / \mathrm{Alg} / \mathrm{CA} / \mathrm{NPK}$ formulations.

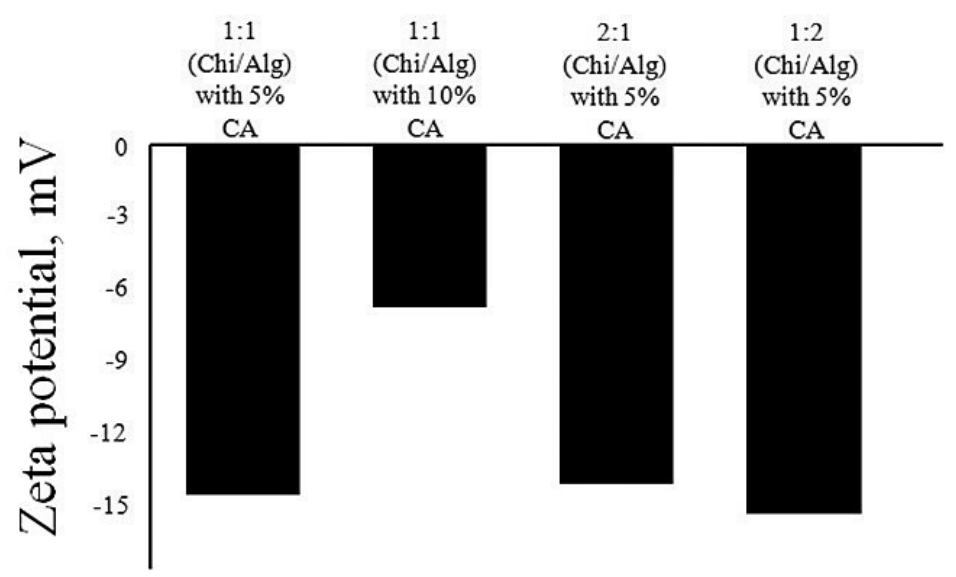

Figure 3. Zeta potential values of various $\mathrm{Chi} / \mathrm{Alg} / \mathrm{CA} / \mathrm{NPK}$ formulations. 
except for the Chi/Alg (2:1) formulation. This would be possible if by any chance the chitosan is distributed inward the dispersion particles upon measurement. Similarly as in the study by Silva and his colleagues (Silva, et al., 2016), the alginate is oriented towards the surface of the multilayer chitosan/alginate nanocapsule. Additionally, the NPK fertilizer utilized in this study is in the form of ions which may cause the phosphate groups interact to the excess chitosan molecules creating a negative surface charge.

\section{FTIR spectroscopy to follow the crosslinking process of the coated chitosan/alginate (Chi/ Alg) NPK fertilizer}

Figure 4 shows the changes in IR spectra as the reaction of $\mathrm{Chi} / \mathrm{Alg} / \mathrm{CA} / \mathrm{NPK}$ (as a crosslinked fertilizer formulation) proceeds to completion from Figure 4A to Figure 4C. Figure 4A consists of alginate-NPK followed by an interaction of calcium-alginate and $\mathrm{Chi} / \mathrm{Alg}$ interaction (Figure 4B) then ended up with citric acid crosslinking process (Figure 4C). The peak at 1086 $\mathrm{cm}^{-1}$ is maintained; however, its intensity decreases as the citric acid crosslinking happens, because the hydroxyl groups are being used for ester formation (Demitri, et al., 2008); thus, some of them are converted to $\mathrm{C}-\mathrm{O}$ stretch in ester resulting in the appearance of band at $1210 \mathrm{~cm}^{-1}$ at the reaction stage $\mathrm{C}$. The changes at $1430 \mathrm{~cm}^{-1} \mathrm{can}$ be attributed to the Chi/Alg interaction as well as the interaction with calcium (Kumar, et al., 2015) and the changes in the broad peak from 3800 to $2500 \mathrm{~cm}^{-1}$ can be accounted for the participation of both polymers in the crosslinking process. Lastly, the appearance of peak $1740 \mathrm{~cm}^{-1}$ indicates an ester formation; thus, a citric acid crosslinking occurs among the Chi/Alg molecules and it is also supported by the shifting of the citric acid peak (Figure 4D).

\section{Fluorescence spectroscopy to evaluate encapsulation efficiency of the chitosan/ alginate (Chi/Alg) materials}

The experiment was done using sodium 8-anilino-1-naphthalenesulfonate (ANS) dye as a fluorescent agent. This dye is slightly soluble in pure water and provides a stronger fluorescence emission in a hydrophobic environment compared to that of hydrophilic one which means that the encapsulation process using $\mathrm{Chi} / \mathrm{Alg}$ can be confirmed if the emission intensity maximizes, as the process proceeds to completion. In Figure 5, the ANS dye has its minimum emission intensity followed by uncrosslinked polymer which is just slightly higher than the emission of the ANS dye

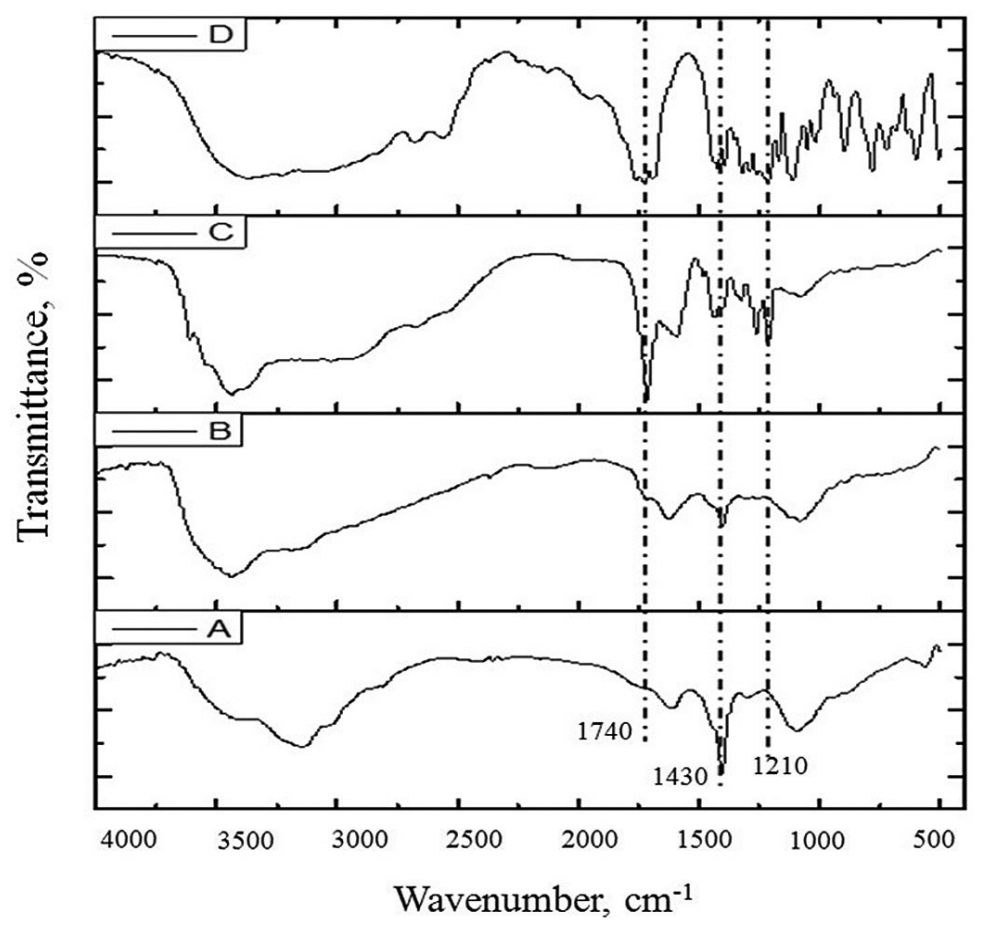

Figure 4. IR spectra of each reaction stage:(A) NPK fertilizer in Alginate solution; (B) Chitosan is added to Alginate and NPK; (C) Citric acid-crosslinked Chitosan/Alginate NPK. The citric acid spectrum is also shown for comparison (D). 
solution; this indicates that the dye is not yet restricted to the hydrophobic part of the polymer. However, after the crosslinking process, there is an abrupt increase in emission intensity which means that the ANS dye is within the domain (Penaloza, et al., 2013) and restricted in the polymer material resulting to extremely large values. Hence, the concept of encapsulation is confirmed.

\section{Scanning electron microscopy of the coated chitosan/alginate (Chi/Alg) NPK fertilizer}

The SEM image of Chi/Alg (1:1) with 10\% $(\mathrm{w} / \mathrm{w})$ citric acid is shown in Figure 6. Most of the particles are globular and fall within the size range observed in the particle size analysis using dynamic light scattering measurements.

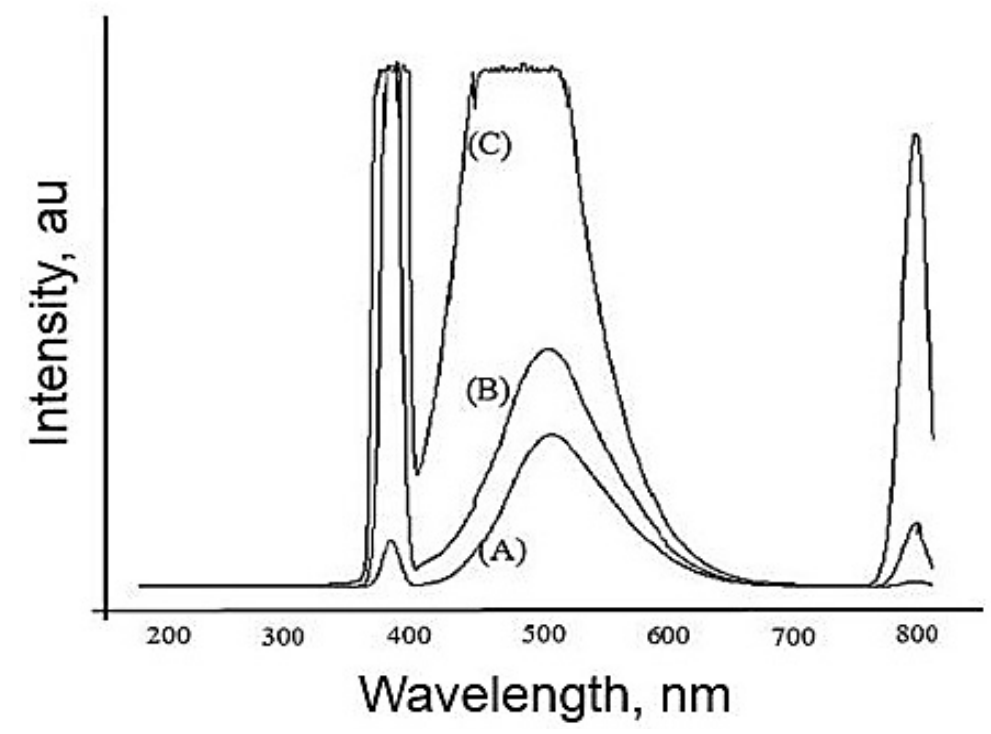

Figure 5. A fluorescent dye, 8-Anilino-1-naphthalenesulfonic acid (ANS), is used as a model active to be encapsulated. The fluorescence measurement of the ANS dye, in (A) water; (B) water containing chitosan/ alginate (before crosslinking) and (C) water containing chitosan/alginate and citric acid (after crosslinking).

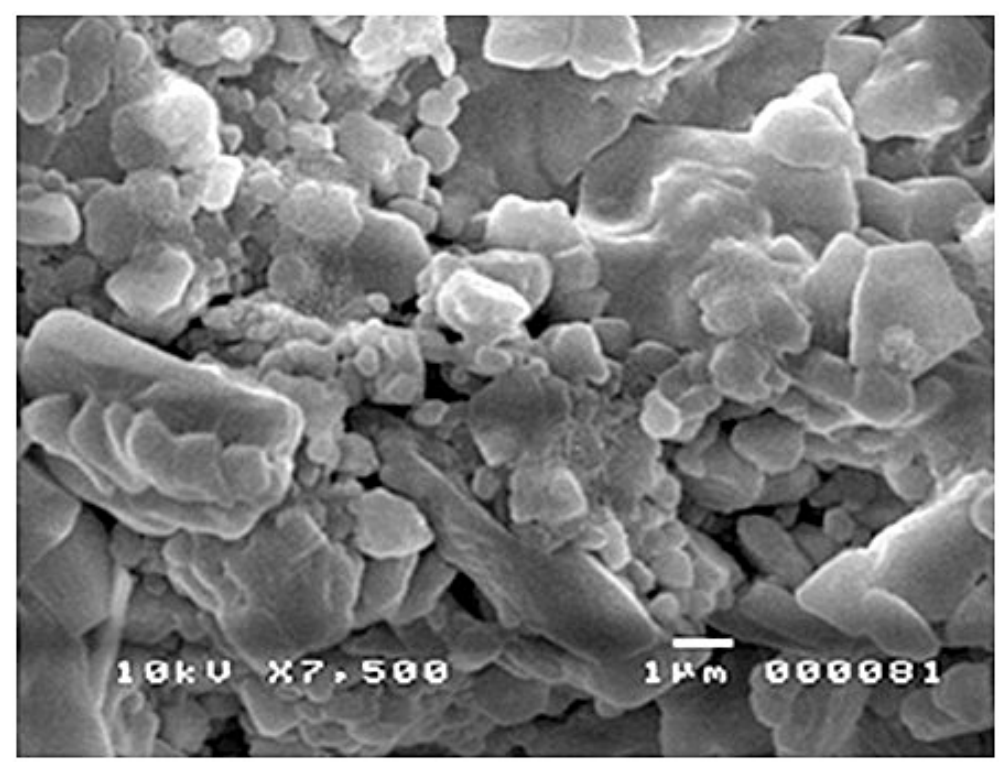

Figure 6. SEM image of the crosslinked polymer (chitosan/alginated reacted with citric acid) containing NPK fertilizer. The bar scale is $1 \mu \mathrm{m}$. 


\section{Release studies of the coated chitosan/ alginate (Chi/Alg) NPK fertilizer under various $\mathrm{pH}$ conditions}

The release profiles of $\mathrm{Chi} / \mathrm{Alg}$ (1:1) with $10 \% \mathrm{w} / \mathrm{w} \mathrm{CA}$ at various $\mathrm{pH}$ levels are shown in Figure 7. It is observed that the Chi/Alg NPK exhibits controlled release behavior. It can be seen by curve inspection that the release rate increases with $\mathrm{pH}$. This demonstrates the solubility of polymer components under slightly alkaline conditions (M. S. Kim, Park, Gu, \& Kim, 2012) and it also demonstrates the resistance of alginate at slightly acidic medium. It was shown in the curve that approximately $40 \%$ of NPK were released for the period of 30 days which is due to the chitosan-alginate interaction in the polyelectrolyte complex (Silva, et al., 2016) and this complies with standard which is less than $70 \%$ for 28 days (C.-W. Liu, Sung, Chen, \& Lai, 2014). The closeness of curves at different $\mathrm{pH}$ levels was also observed; the reason for this is due to the solubility of chitosan under slightly acidic conditions
(Kumar, et al., 2015) that leads to the release of some entrapped nutrients in the matrix.

The release profiles for each nutrient at various $\mathrm{pH}$ values were fitted into Korsemeyer-Peppas model. Upon linearization of the said model, it generates an equation of the line in which y is log release, $\mathrm{x}$ is log time, $\mathrm{y}$-intercept is log release rate constant, and slope is the release exponent, $\mathrm{n}$ which defines the release mechanism (Shariff, Pk, Klk, \& M, 2007; Wang, et al., 2017). On the basis of Figure 7, it can be seen that all nutrients and $\mathrm{pH}$ levels have slope that lies between 0.43 and 0.85 , which is indication of both diffusion controlled release and matrix-based release $(\mathrm{Li}$, et al., 2008). In addition, the values of y-intercept increase with $\mathrm{pH}$; hence, it supports the profile that release is highest at basic condition because of the solubility of the matrix.

Fertilizer efficacy evaluation of the coated chitosan/alginate (Chi/Alg) NPK fertilizer

In the first few weeks, the second, third and fourth setup continuously grows and their growth
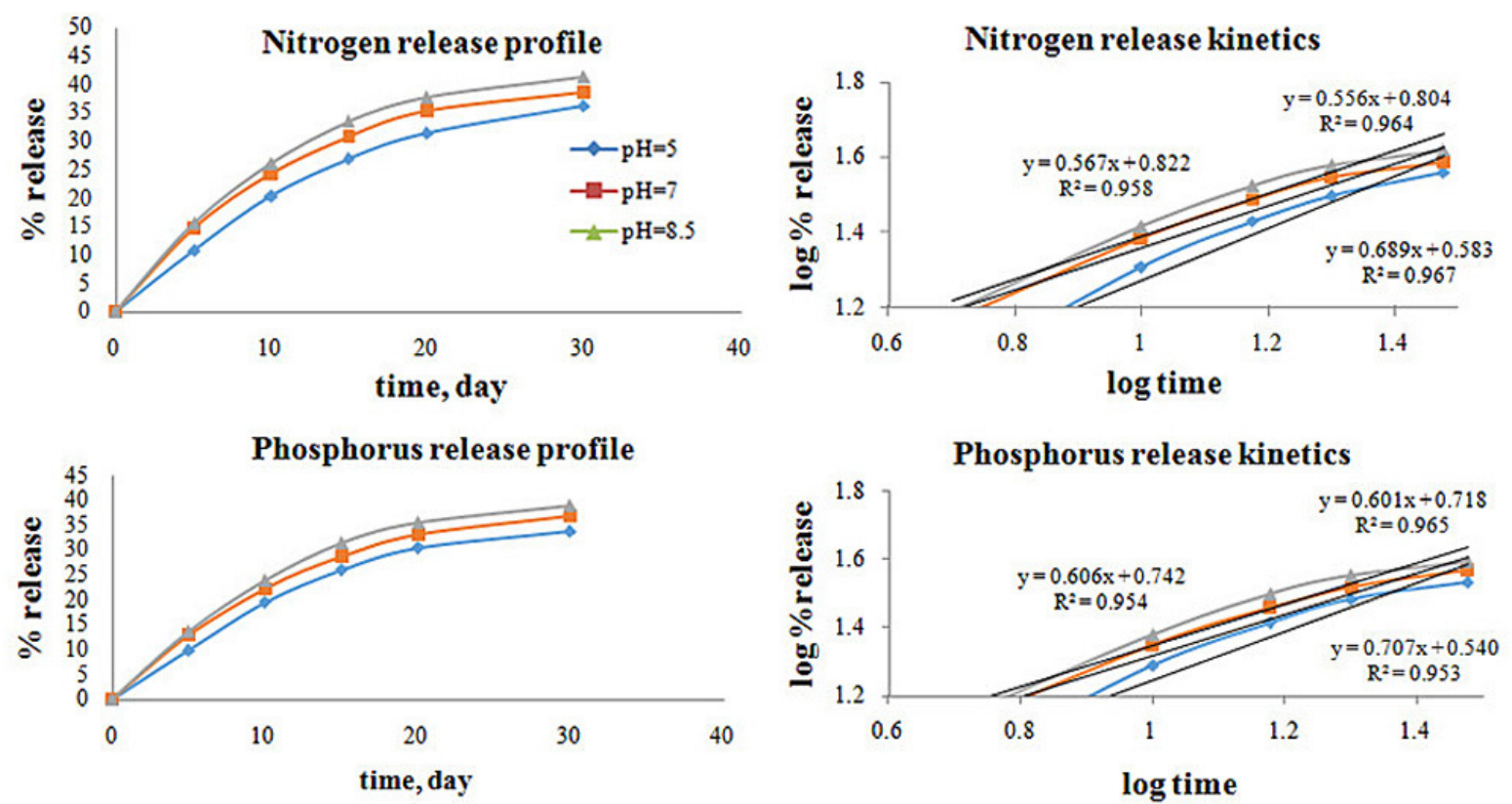

Potassium release profile
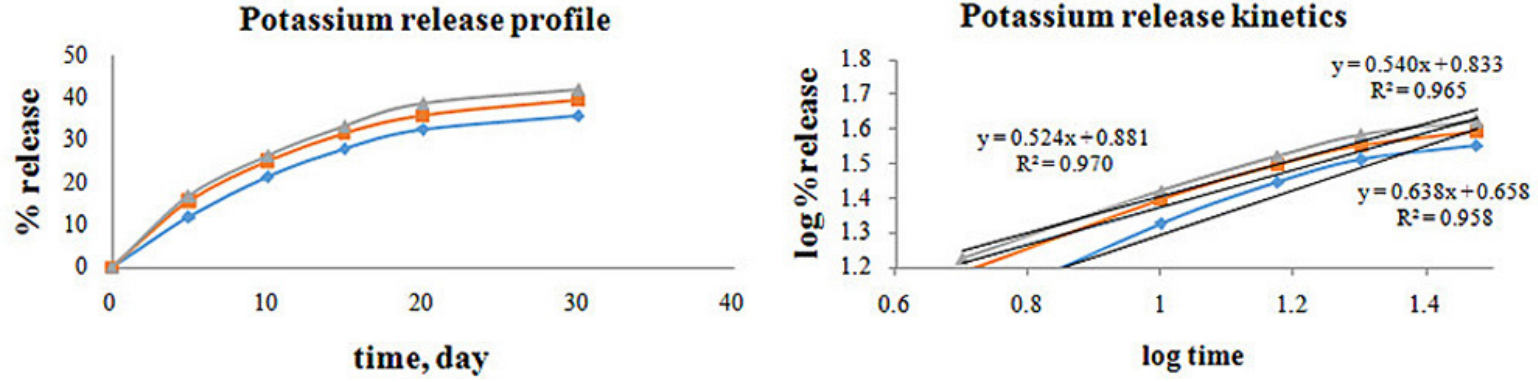

Figure 7. Release profiles (left) and kinetics (right) of Chitosan/Alginate (1:1) NPK with 10\% (w/w) Citric acid at $\mathrm{pH} 5.00$ (diamond plots), 7.00 (square plots), and 8.5 (triangle plots). 
parameters (plant height, leaf length and leaf width) are seemingly identical. However, as the time goes by, the setup which contains the Chi/ Alg NPK was continuously growing. It was also observed that the second setup growth slowed down due to the frequent rains (the trial was made during rainy season), where because of this, nutrients can be lost through run off (Saigusa, et al., 2001). Figure 8 shows that the Chi/Alg coated fertilizer produces observable increase in terms of plant growth parameters after 8 weeks; this may be related to its release kinetics as described in the previous data. This may indicate that the controlled release is working on the given condition especially wherein frequent rains are observed which causes leaching. Hence, the controlled release property was observed.

\section{CONCLUSION}

The coated NPK fertilizer based on citric acid-crosslinked chitosan/alginate (Chi/Alg/CA/ NPK) was successfully prepared as colloidal dispersion. The highest encapsulation efficiency, which ranges from $85-89 \%$, was observed in 1:1 Chi/Alg ratio with $10 \%$ citric acid (per weight polymer) formulation. A SEM image revealed the existence of spherical particles, which supports particle size distribution and encapsulation phenomenon. FTIR showed successful citric acid crosslinking and interaction of individual components. Moreover, fluorescence spectroscopy confirms the existence of the encapsulation process. The encapsulated NPK formulation demonstrated the controlled release behavior at various $\mathrm{pH}$ levels and followed the Korsemeyer-Peppas model;
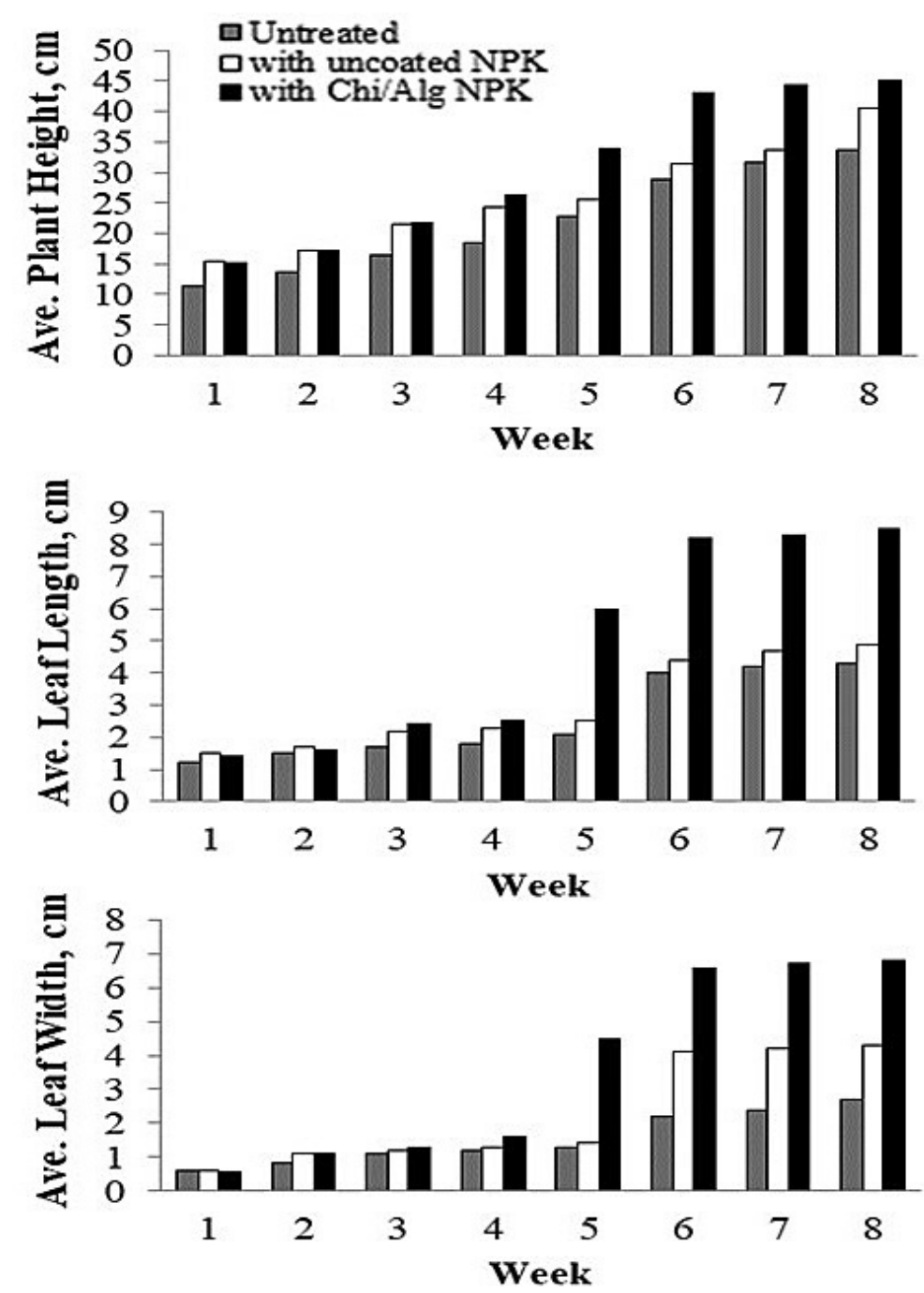

Figure 8. Growth parameters of Mung bean studied for eight weeks in various setups: Untreated (gray); with uncoated NPK fertilizer (white) and; with Chi/Alg coated NPK fertilizer (black). Plant height (Top); Leaf length (Middle) and; Leaf width (Bottom). 
this shows that the release is both governed by polymer relaxation and diffusion. Lastly, these colloidal dispersions showed a good controlled release performance in the pot experiment and hence can be used in wide agricultural setting.

\section{Acknowledgment}

The De La Salle University is acknowledged for the scholarship support extended to Vince St. D. Mesias through De La Salle University Graduate Scholarship-Financial Assistance. The authors also would like to acknowledge the technical and chemical assistance extended by the D\&L Industries, Inc. and Chemrez.

\section{REFERENCES}

1. Araújo B.R., Romão L.P.C., Doumer M.E., \& Mangrich, A.S. 2017. Evaluation of the interactions between chitosan and humics in media for the controlled release of nitrogen fertilizer. Journal of Environmental Management, 190, 122-131.

2. Awadhiya A., Kumar D., \& Verma V. 2016. Crosslinking of agarose bioplastic using citric acid. Carbohydrate Polymers, 151, 60-67.

3. Azeem B., KuShaari K., Man Z.B., Basit A., \& Thanh T.H. 2014. Review on materials \& methods to produce controlled release coated urea fertilizer. Journal of Controlled Release, 181, 11-21.

4. Baysal K., Aroguz A.Z., Adiguzel Z., \& Baysal B.M. 2013. Chitosan/alginate crosslinked hydrogels: Preparation, characterization and application for cell growth purposes. International Journal of Biological Macromolecules, 59, 342-348.

5. Bortolin A., Aouada F.A., De Moura M.R., Ribeiro C., Longo E., \& Mattoso L.H.C. 2012. Application of polysaccharide hydrogels in adsorption and controlled-extended release of fertilizers processes. Journal of Applied Polymer Science, 123(4), 2291-2298.

6. Bortolin A., Aouada F.A., Mattoso L.H.C., \& Ribeiro C. 2013. Nanocomposite PAAm/methyl cellulose/montmorillonite hydrogel: evidence of synergistic effects for the slow release of fertilizers. Journal of Agricultural and Food Chemistry, 61(31), 7431-7439.

7. Brown J.Q., \& McShane M.J. 2006. Modeling of spherical fluorescent glucose microsensor systems: Design of enzymatic smart tattoos. Biosensors and Bioelectronics, 21(9), 1760-1769.

8. Campos E. V.R., de Oliveira J.L., Fraceto L.F., \& Singh B. 2015. Polysaccharides as safer release systems for agrochemicals. Agronomy for Sustainable Development, 35(1), 47-66.
9. Chen C., Gao Z., Qiu X., \& Hu S. 2013. Enhancement of the controlled-release properties of chitosan membranes by crosslinking with suberoyl chloride. Molecules, 18(6), 7239-7252.

10. Das R.K., Kasoju N., \& Bora U. 2010. Encapsulation of curcumin in alginate-chitosan-pluronic composite nanoparticles for delivery to cancer cells. Nanomedicine: Nanotechnology, Biology, and Medicine, 6(1), 153-160.

11. Dasgupta Q., Madras G., \& Chatterjee K. 2016. Controlled release kinetics of p-aminosalicylic acid from biodegradable crosslinked polyesters for enhanced anti-mycobacterial activity. Acta Biomaterialia, 30, 168-176.

12. Dash M., Chiellini F., Ottenbrite R.M., \& Chiellini E. 2011. Chitosan -A versatile semi-synthetic polymer in biomedical applications. Progress in Polymer Science (Oxford), 36(8), 981-1014.

13. de Cuadro P., Belt T., Kontturi K.S., Reza M., Kontturi E., Vuorinen T., \& Hughes M. 2015. Cross-linking of cellulose and poly(ethylene glycol) with citric acid. Reactive and Functional Polymers, 90, 21-24.

14. Demitri C., Del Sole R., Scalera F., Sannino A., Vasapollo G., Maffezzoli A., Ambrosio L. \& Nicolais L. 2008. Novel superabsorbent cellulose-based hydrogels crosslinked with citric acid. Journal of Applied Polymer Science, 110(4), 2453-2460.

15. Essawy H.A., Ghazy M.B.M., El-Hai F.A., \& Mohamed M.F. 2016. Superabsorbent hydrogels via graft polymerization of acrylic acid from chitosancellulose hybrid and their potential in controlled release of soil nutrients. International Journal of Biological Macromolecules, 89, 144-151.

16. Ghorpade V.S., Yadav A.V., \& Dias R.J. 2016. Citric acid crosslinked cyclodextrin/hydroxypropylmethylcellulose hydrogel films for hydrophobic drug delivery. [Article]. International Journal of Biological Macromolecules, 93, 75-86.

17. Gombotz W.R., \& Wee S.F. 1998. Protein release from alginate matrices. Advanced Drug Delivery Reviews, 31(3), 267-285.

18. Habibi N., Kamaly N., Memic A., \& Shafiee H. 2016. Self-assembled peptide-based nanostructures: Smart nanomaterials toward targeted drug delivery. Nano Today, 11(1), 41-60.

19. Hori K., Penaloza D.P., Shundo A., \& Tanaka K. 2012. Time-dependent heterogeneity in viscoelastic properties of worm-like micelle solutions. Soft Matter, 8(28), 7361-7364.

20. Hu B., \& Huang Q.R. 2013. Biopolymer based nano-delivery systems for enhancing bioavailability of nutraceuticals. Chinese Journal of Polymer Science (English Edition), 31(9), 1190-1203.

21. Jamnongkan T., \& Kaewpirom S. 2010. Potassium release kinetics and water retention of controlled-release fertilizers based on chitosan hydrogels. Journal of Polymers and the Environment, 18(3), 413-421. 
22. Jarosiewicz A., \& Tomaszewska M. 2003. Controlled-release NPK fertilizer encapsulated by polymeric membranes. Journal of Agricultural and Food Chemistry, 51(2), 413-417.

23. Kim H.J., Koo J. M., Kim S.H., Hwang S.Y., \& Im S.S. 2017. Synthesis of super absorbent polymer using citric acid as a bio-based monomer. Polymer Degradation and Stability, 144, 128-136.

24. Kim M.S., Park S.J., Gu B.K., \& Kim C.-H. 2012. Ionically crosslinked alginate-carboxymethyl cellulose beads for the delivery of protein therapeutics. Applied Surface Science, 262, 28-33.

25. Krishna Sailaja A., Amareshwar P., \& Chakravarty P. 2011. Different techniques used for the preparation of nanoparticles using natural polymers and their application. International Journal of Pharmacy and Pharmaceutical Sciences, 3(SUPPL. 2), 45-50.

26. Kumar S., Chauhan N., Gopal M., Kumar R., \& Dilbaghi N. 2015. Development and evaluation of alginate-chitosan nanocapsules for controlled release of acetamiprid. International Journal of Biological Macromolecules, 81, 631-637.

27. Li P., Dai Y.-N., Zhang J.-P., Wang A.-Q., \& Wei Q. 2008. Chitosan-alginate nanoparticles as a novel drug delivery system for nifedipine. International journal of biomedical science : IJBS, 4(3), 221-228.

28. Lima A.C., Song W., Blanco-Fernandez B., Alvarez-Lorenzo C., \& Mano J. F. 2011. Synthesis of temperature-responsive Dextran-MA/PNIPAAm particles for controlled drug delivery using superhydrophobic surfaces. Pharmaceutical Research, 28(6), 1294-1305.

29. Liu C.-W., Sung Y., Chen B.-C., \& Lai H.-Y. 2014. Effects of nitrogen fertilizers on the growth and nitrate content of lettuce (Lactuca sativa L.). International journal of environmental research and public health, 11(4), 4427-4440.

30. Liu Y., Shen X., Zhou H., Wang Y., \& Deng L. 2016. Chemical modification of chitosan film via surface grafting of citric acid molecular to promote the biomineralization. Applied Surface Science, 370, 270-278.

31. Ma M., Tan L., Dai Y., \& Zhou J. 2013. An investigation of flavor encapsulation comprising of regenerated cellulose as core and carboxymethyl cellulose as wall. Iranian Polymer Journal, 22(9), 689-695.

32. Maitra J., \& Shukla V.K. 2014. Cross-linking in hydrogels - a review. American Journal of Polymer Science, 4(2), 25-31.

33. Mali K.K., Dhawale S.C., \& Dias R.J. 2017. Synthesis and characterization of hydrogel films of carboxymethyl tamarind gum using citric acid. International Journal of Biological Macromolecules, 105, 463-470.
34. Monteiro O.A.C., \& Airoldi C. 1999. Some studies of crosslinking chitosan-glutaraldehyde interaction in a homogeneous system. International Journal of Biological Macromolecules, 26(2-3), 119-128.

35. Ni B., Liu M., Lü S., Xie L., \& Wang Y. 2011. Environmentally friendly slow-release nitrogen fertilizer. Journal of Agricultural and Food Chemistry, 59(18), 10169-10175.

36. Oryan A., Kamali A., Moshiri A., Baharvand H., \& Daemi H. 2018. Chemical crosslinking of biopolymeric scaffolds: Current knowledge and future directions of crosslinked engineered bone scaffolds. International Journal of Biological Macromolecules, 107(PartA), 678-688.

37. Penaloza D.P., Shundo A., Matsumoto K., Ohno M., Miyaji K., Goto M., Tanaka K. 2013. Spatial heterogeneity in the sol-gel transition of a supramolecular system. Soft Matter, 9(21), 5166-5172.

38. Picone C.S.F., \& Cunha R.L. 2013. Chitosan-gellan electrostatic complexes: influence of preparation conditions and surfactant presence. Carbohydrate Polymers, 94(1), 695-703.

39. Qu J., Zhao X., Ma P.X., \& Guo B. 2017. pH-responsive self-healing injectable hydrogel based on $\mathrm{N}$-carboxyethyl chitosan for hepatocellular carcinoma therapy. Acta Biomaterialia, 58, 168-180.

40. Ren L., Zhang Y., Wang Q., Zhou J., Tong J., Chen D., \& Su X. 2018. Convenient method for enhancing hydrophobicity and dispersibility of starch nanocrystals by crosslinking modification with citric acid. International Journal of Food Engineering, 14(4).

41. Riyajan S.-A., \& Nuim J. 2013. Interaction of green polymer blend of modified sodium alginate and carboxylmethyl cellulose encapsulation of turmeric extract. International Journal of Polymer Science, 2013, 10.

42. Rodríguez-Velázquez E., Alatorre-Meda M., \& Mano J.F. 2015. Polysaccharide-based nanobiomaterials as controlled release systems for tissue engineering applications. Current Pharmaceutical Design, 21(33), 4837-4850.

43. Rowley J.A., Madlambayan G., \& Mooney D. J. 1999. Alginate hydrogels as synthetic extracellular matrix materials. Biomaterials, 20(1), 45-53.

44. Roy A., Singh S. K., Bajpai J., \& Bajpai A. K. 2014. Controlled pesticide release from biodegradable polymers. Central European Journal of Chemistry, 12(4), 453-469.

45. Saigusa M., Kikuchi Y., Takei N., Kanazawa S., Kasugai A., Sakamoto K., \& Imai T. 2001. The evaluation of mode purity for ECCD using single deep grooved polarizer. Fusion Engineering and Design, 53(1), 505-510. 
46. SaraydIn D., Karadağ E., \& Güven O. 2000. Relationship between the swelling process and the releases of water soluble agrochemicals from radiation crosslinked acrylamide/itaconic acid copolymers. Polymer Bulletin, 45(3), 287-294.

47. Seiffert S., Thiele J., Abate A.R., \& Weitz D.A. 2010. Smart microgel capsules from macromolecular precursors. Journal of the American Chemical Society, 132(18), 6606-6609.

48. Shariff A., Pk M., Klk P., \& M M. 2007. Entrapment of andrographolide in cross-linked alginate pellets: I. Formulation and evaluation of associated release kinetics. Pakistan Journal of Pharmaceutical Sciences, 20(1), 1-9.

49. Shi R., Bi J., Zhang Z., Zhu A., Chen D., Zhou X., Zhang L., \& Tian W. 2008. The effect of citric acid on the structural properties and cytotoxicity of the polyvinyl alcohol/starch films when molding at high temperature. Carbohydrate Polymers, 74(4), 763-770.

50. Shundo A., Hoshino Y., Higuchi T., Matsumoto Y., Penaloza D.P., Matsumoto, K., Ohono M., Miyaji K., Goto M. \& Tanaka K. 2014. Facile microcapsule fabrication by spray deposition of a supramolecular hydrogel. RSC Advances, 4(68), 36097-36100.

51. Shundo A., Penaloza D.P., \& Tanaka K. 2013. Microscopic heterogeneity in viscoelastic properties of molecular assembled systems. Chinese Journal of Polymer Science (English Edition), 31(1), 1-11.

52. Silva D., Pinto L.F.V., Bozukova D., Santos L.F., Serro A.P., \& Saramago B. 2016. Chitosan/alginate based multilayers to control drug release from ophthalmic lens. Colloids and Surfaces B: Biointerfaces, 147, 81-89.

53. Stulzer H.K., Tagliari M.P. Parize, A.L. Silva, M. A.S., \& Laranjeira M.C.M. 2009. Evaluation of cross-linked chitosan microparticles containing acyclovir obtained by spray-drying. Materials Science and Engineering: C, 29(2), 387-392.

54. Sultana K., Godward G., Reynolds N., Arumugaswamy R., Peiris P., \& Kailasapathy K. 2000. Encapsulation of probiotic bacteria with alginate-starch and evaluation of survival in simulated gastrointestinal conditions and in yoghurt. International Journal of Food Microbiology, 62(1-2), 47-55.

55. Tan W.H., \& Takeuchi S. 2007. Monodisperse alginate hydrogel microbeads for cell encapsulation. Advanced Materials, 19(18), 2696-2701.

56. Wang Y., Papadimitrakopoulos F., \& Burgess D.J. 2013. Polymeric "smart" coatings to prevent foreign body response to implantable biosensors. Journal of Controlled Release, 169(3), 341-347.

57. Wang Y., Wang J., Yuan Z., Han H., Li T., Li L.,\& Guo X. 2017. Chitosan cross-linked poly(acrylic acid) hydrogels: Drug release control and mechanism. Colloids and Surfaces B: Biointerfaces, 152, 252-259.

58. Xiao X., Zhou H., \& Qian K. 2017. Mechanism study of biopolymer hair as a coupled thermo-water responsive smart material. Smart Materials and Structures, 26(3), 035023

59. Zhang Y., Wei W., Lv P., Wang L., \& Ma G. 2011. Preparation and evaluation of alginate-chitosan microspheres for oral delivery of insulin. European Journal of Pharmaceutics and Biopharmaceutics, 77(1), 11-19.

60. Zhao X., Yim C.H., Du N., \& Abu-Lebdeh Y. 2018. Crosslinked chitosan networks as binders for silicon/graphite composite electrodes in Li-Ion batteries. Journal of the Electrochemical Society, 165(5), A1110-A1121. 